\title{
Ankle Sprain Has Higher Occurrence During the Latter Parts of Matches: Systematic Review With Meta-Analysis
}

\author{
Marcos de Noronha, Eleisha K. Lay, Madelyn R. Mcphee, George Mnatzaganian, \\ and Guilherme S. Nunes
}

\begin{abstract}
Context: Ankle sprains are common injuries in sports, but it is unclear whether they are more likely to occur in a specific period of a sporting game. Objective: To systematically review the literature investigating when in a match ankle sprains most likely occurred. Evidence Acquisition: The databases CINAHL, EMBASE, MEDLINE, and SPORTDiscus were searched up to August 2016, with no restriction of date or language. The search targeted studies that presented data on the time of occurrence of ankle sprains during sports matches. Data from included studies were analyzed as a percentage of ankle sprain occurrence by halftime and by quarters. Meta-analyses were run using a random effects model. The quality assessment tool for quantitative studies was used to assess the article's quality. Evidence Synthesis: The searches identified 1142 studies, and 8 were included in this review. A total of 500 ankle sprains were reported during follow-up time, which ranged from 1 to 15 years, in 5 different sports (soccer, rugby, futsal, American football, and Gaelic football). The meta-analyses, including all 8 studies, showed that the proportion of ankle sprains during the first half $(0.44 ; 95 \%$ confidence interval $[\mathrm{CI}], 0.38-0.50)$ was smaller than the second half $(0.56 ; 95 \% \mathrm{CI}, 0.50-0.62)$. For the analyses by quarters, the proportion of ankle sprains in the first quarter $(0.14 ; 95 \%$ CI, $0.09-$ $0.19)$ was considerably smaller than the second $(0.28$; $95 \%$ CI, $0.24-0.32)$, third $(0.25$; 95\% CI, 0.17-0.34), and fourth (0.29; 95\% CI, 0.22-0.36) quarters. Conclusion: The results of this review indicate that ankle sprains are more likely to occur later in the game during the second half or during the latter minutes of the first half.
\end{abstract}

Keywords: soccer, rugby, fatigue, prevalence, lower limbs

Ankle sprains are one of the most common injuries experienced by individuals involved in physical activity, accounting for approximately $80 \%$ of ankle injuries. ${ }^{1,2}$ They are the most frequent sports-related injury, particularly in team sports that demand repeated direction change, jumping, pivoting, and challenging landing postures. ${ }^{3}$ Of those sprains, approximately $77 \%$ are lateral ankle sprains. ${ }^{2}$ The most described mechanism of injury involves a combination of inversion, plantarflexion, and internal rotation, ${ }^{4}$ although there is some debate on whether the ankle needs to be in plantarflexion for an ankle sprain to occur. ${ }^{5}$ Nevertheless, ankle sprains can severely damage ligaments and muscles. ${ }^{6}$

A systematic review reported that ankle sprains account for $11.2 \%$ to $20.8 \%$ of all injuries in sports, making this the most common injury in 24 sports (70 sports modalities investigated). ${ }^{1}$ Therefore, such high prevalence has an impact on not only the athlete but also the sporting team, organization, and the wider community. For the athlete, regardless of whether they are an elite or recreational player, ankle injury causes pain, loss of playing time, loss of fitness, potentially time off work, and medical expenditures. ${ }^{1}$ At an elite level, absence of an athlete due to an unexpected injury can result in defeats in major games, financial losses, and huge economic burden. ${ }^{1}$ At a recreational level, participation in sport can be affected, with consequences in fitness and social interaction. ${ }^{7,8}$ It has been stated that a quarter of people who suffer an ankle sprain are unable to attend school or work for more

de Noronha, Mcphee, and Mnatzaganian are with Rural Health School, La Trobe University, Bendigo, Victoria, Australia. Lay is with Fusion Physiotherapy, Bendigo, Victoria, Australia. Nunes is with the Universidade Federal de São Carlos, São Carlos, São Paulo, Brazil. de Noronha (M.DeNoronha@latrobe.edu.au) is corresponding author. than 7 days after the injury. ${ }^{8}$ Furthermore, Andrew et $\mathrm{al}^{7}$ found at 12-months postinjury participants reported a significant reduction in their physical activity levels, with similar mean reductions in those who reported a full recovery compared with those who did not fully recover. Finally, a large burden is placed on the health care system as a consequence of ankle sprains. ${ }^{8}$

So, why do ankle sprains occur? Many intrinsic risk factors have been investigated, including history of ankle sprain, weight, height, joint laxity, functional instability, and balance. ${ }^{9-11}$ There are also numerous extrinsic risk factors that influence the risk of sprain, including the surface on which the sport is played and the general environment. There is also developing evidence that suggests that fatigue is a key extrinsic risk factor. It is postulated that greater mechanical demands placed on a joint in a fatigued state decrease muscle coordination and activation, causing inadequate joint stabilization. ${ }^{12}$ This is in line with the fact that overall injury rates have been shown to increase toward the end of matches. ${ }^{2,13,14}$ Overall, it seems plausible to consider the possibility that in a fatigued state, proprioception and postural stability will be impaired and will consequently increase the risk of an ankle sprain. ${ }^{15}$ Conversely, inadequate warm-ups at the beginning of a game and after long breaks have been shown to increase the risk of injuries. ${ }^{16,17}$ However, this data are not specific to ankle sprains; therefore, a gap on the knowledge remains.

So it seems that lack of preparation (warm-up) could lead to injuries, including ankle sprains, and one may question whether ankle sprains would more likely be seen in the first stages of a match, while tissues are not yet warmed up. However, if fatigue is a stronger risk factor for ankle sprains, perhaps it should be expected that ankle sprains would be happening at a later stage in a match, when a fatigue stage has been reached by players at risk. Nevertheless, not many studies have been set up to identify when during a 
match ankle sprains are most likely to occur. The current literature shows some studies that have recorded data, which can be used to answer that question; however, most studies show data on time of ankle sprain occurrence as only complementary information and tend to be underpowered. Therefore, the purpose of this study was to use the technique of meta-analysis to synthesize the existing literature to determine when in a game or match ankle sprains most frequently occurred.

\section{Evidence Acquisition}

The review protocol for this systematic review was registered at PROSPERO (CRD42016047169).

\section{Identification and Selection of Studies}

A systematic literature search of CINAHL, EMBASE, MEDLINE, and SPORTDiscus was conducted from inception to August 2016. The search strategy outlined for MEDLINE is described in Table 1. Adjustments to this search strategy were made as necessary to search other databases. There was no date or language restriction in the search. Two reviewers independently screened titles, abstracts, and full texts for eligibility against inclusion/exclusion criteria, and disagreements were resolved via discussion. Full-text articles were

\section{Table 1 Search Strategy Implemented for MEDLINE}

\begin{tabular}{|c|c|}
\hline 1 & Ankle/ \\
\hline 2 & Ankle joint/ \\
\hline 3 & ankle \\
\hline 4 & Talocrural joint \\
\hline 5 & Or/1-4 \\
\hline 6 & "Sprains and Strains"/ \\
\hline 7 & Sprain* \\
\hline 8 & Strain* \\
\hline 9 & Rupture* \\
\hline 10 & Tear \\
\hline 11 & Torn \\
\hline 12 & Or/6-11 \\
\hline 13 & 5 AND 12 \\
\hline 14 & Ankle injuries/ \\
\hline 15 & 13 or 14 \\
\hline 16 & Epidemiology/ \\
\hline 17 & Epidemiology* \\
\hline 18 & Survey \\
\hline 19 & Incidence/ \\
\hline 20 & Incidence* \\
\hline 21 & Prevalence/ \\
\hline 22 & Prevalence* \\
\hline 23 & Or/16-22 \\
\hline 24 & Game* \\
\hline 25 & Match \\
\hline 26 & Sports/ \\
\hline 27 & Sport* \\
\hline 28 & Or/24-27 \\
\hline 29 & 15 AND 23 AND 28 \\
\hline
\end{tabular}

obtained, and authors were contacted when clear data were not presented regarding the number of ankle sprains to provide clarification of these results and determine if the studies could be included in the review. Studies were included if there was data presented for the number of ankle sprains and the time the ankle sprain occurred during a sports game or match and if the population was healthy adults. Studies were excluded if data on ankle sprains were combined with other injuries.

\section{Evaluation of Methodological Quality}

The methodological quality of the included studies was assessed using the quality assessment tool for quantitative studies (QATQS) by the Effective Public Health Practice project. ${ }^{18}$ The tool has been judged to have fair-to-excellent interrater reliability ${ }^{19}$ together with excellent intrarater reliability and acceptable construct validity. ${ }^{20}$ The quality assessment tool contains 21 items categorized into 8 subgroups. The first 6 subgroups-selection, study design, confounders, blinding, data collection, and withdrawals/dropoutscontribute to the overall score. The tool screens for selection, allocation, confounding, detection, and attrition bias. The quality assessment tool was independently applied by 2 reviewers to all included studies, with disagreements resolved via consensus. Studies were judged to be of high quality if they did not receive a judgment of "weak" for any of the first 6 subgroups. Papers that received 1 "weak" allocation were considered to be of moderate quality, and studies receiving 2 or more "weak" ratings were considered to be of low quality.

\section{Data Extraction and Analysis}

Data extracted from studies included the number and age of the participants, sport played, duration of the match, and the time that the ankle sprain occurred. Data were analyzed by organizing the data by time of ankle sprain. The analyses were conducted by halves, quarters, and 15-minute time periods when possible; we also analyzed data by sport when more than 1 study investigated the same sport. Pooled injury proportions (with 95\% confidence intervals) in either each half, each quarter, or each 15-minute period were separately calculated using a random effects model based on the DerSimonian and Laird method. ${ }^{20}$ This method incorporates an estimate of the between-study variation into both the study weights and the standard error of the estimate of the common effect. The precision of an estimate from each included study was represented by the inverse of the variance of the outcome pooled across all studies. If the value of the pooled proportion (either subtotal or overall) and its 95\% confidence interval does not include zero, then the effect size was statistically significant at the 5\% level $(P<.05)$. We also estimated the differences in the proportion of ankle sprains between halves, quarters, and 15 -minute periods at the $5 \%$ level $(P<.05)$. The heterogeneity among included studies was assessed using the $\mathrm{I}^{2}$ statistic. $\mathrm{I}^{2}$ values of below $25 \%$ indicate low heterogeneity; $25 \%$ to $75 \%$, moderate heterogeneity; and $75 \%$ or more, high heterogeneity. ${ }^{21}$

\section{Evidence Synthesis}

\section{Study Selection}

The process of the literature search and selection of articles are presented in Figure 1. The search identified 1142 articles after removal of duplicates. Following screening by 2 independent 


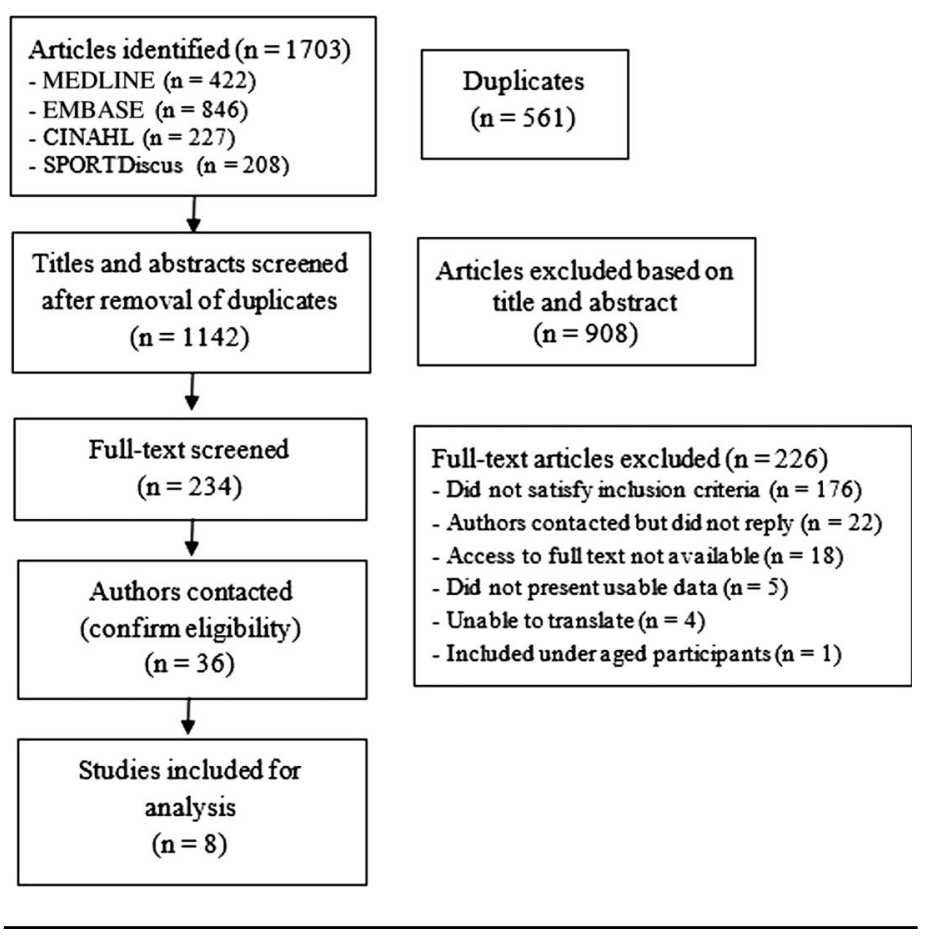

Figure 1 - Flow diagram for study selection.

reviewers, 36 studies were identified as potential inclusions. Authors of the 36 papers were contacted either to confirm eligibility or to request further details, and 14 authors replied to our contact. Eight studies were found to satisfy the inclusion criteria (Table 2).

\section{Study Characteristics}

The total sample size of participants was 3773 (all of them male), excluding participants from the studies Osbahr et $\mathrm{al}^{28}$ and Junge and Dvorak ${ }^{27}$ because these papers did not state the total number of included participants. Of the 8 papers included in this systematic review, the sports analyzed were soccer, rugby union, Gaelic football, American football (National Football League), and futsal. The shortest game time was 40 minutes (futsal), and the longest game time was 90 minutes (soccer). A total of 500 sprains were reported in a follow-up time ranging from 1 to 15 years (Table 2).

\section{Methodological Quality}

The QATQS resulted in 2 strong, ${ }^{22,24} 3$ moderate, ${ }^{23,27,28}$ and 3 weak $25,26,29$ articles (Table 3). All of the studies satisfied the QATQS item data collection, and 6 of the 8 papers satisfied the QATQS item selection criteria, meaning the population studied is an accurate representation of the target population. However, given the nature of the study designs, none of the papers satisfied the QATQS item blinding assessors, and it was difficult to conclude if participants were appropriately blinded. Another item that was unsatisfied was confounders, with only 2 of the 8 papers reporting the characteristics of their participants (Table 3 ).

\section{Meta-Analysis by Halves}

The proportion of ankle sprains during the first half of the matches was lower compared with the second half $(P=.01$; Figure 2$)$. Heterogeneities for data pooled by halves and overall were moderate ( $\mathrm{I}^{2}=26 \%$ for the first half, $26 \%$ for the second half, and $48 \%$ for overall; Figure 2).

\section{Meta-Analysis by Quarters}

For the meta-analysis by quarters, we transformed the data from studies that presented ankle sprains by intervals of 15 minutes ${ }^{24-26}$ into halves. To do so, we divided the number of sprains that occurred in the mid-15-minute interval in each half by 2 and added that value to each of the quarters in the respective halves. Figure 3 shows that the proportion of ankle sprains seen during the first quarter was lower compared with the second $(P=.01)$, third $(P=$ $.05)$, and fourth quarters $(P=.01)$. There was no difference regarding the proportion of ankle sprains among the second, third, and fourth quarters $(P>.05$; Figure 3$)$. Heterogeneities for data pooled in the first, third, and fourth quarter were moderate $\left(\mathrm{I}^{2}=35 \%\right.$ for the first quarter, $71 \%$ for the third quarter, and $54 \%$ for fourth quarter), and heterogeneity for data pooled in the second quarter was low $\left(\mathrm{I}^{2}=0 \%\right)$. Heterogeneity for the overall pooled data was also moderate $\left(\mathrm{I}^{2}=66 \%\right)$.

\section{Meta-Analysis by Sports}

The proportion of ankle sprain in soccer and rugby was presented by more than 1 study. A meta-analysis was conducted pooling the results of 2 studies ${ }^{22,23}$ that reported the proportion of ankle sprain in rugby $\left(\mathrm{I}^{2}=84.8 \%\right)$, and there were no differences in proportions of ankle sprains among the quarters of the rugby matches $(P>.05)$. Another meta-analysis was conducted pooling the results of 3 studies, ${ }^{24-26}$ which reported the proportion of ankle sprain in soccer by blocks of 15 minutes $\left(\mathrm{I}^{2}=60.2 \%\right)$, and there were also no differences among the 15-minute periods $(P>.05)$.

\section{Discussion}

The results of this review indicate that ankle sprains are more likely to occur later in the game during the second half or during the latter minutes (second quarter) of the first half. Although the reasons for such a pattern are unclear, one possible explanation may be related to the effects of fatigue and consequent increase in the risk of an ankle sprain.

Previous studies have investigated the effects of fatigue on various aspects of the ankle. ${ }^{12,30,31}$ One aspect investigated is the effect of fatigue on proprioception of the ankle, particularly force sense. ${ }^{31}$ Wright and Arnold ${ }^{31}$ studied the effect of a fatigue protocol on force sense in 64 people (32 with functional ankle instability and 32 with no history of ankle sprain) and reported that for all participants fatigue decreased their ability to sense force at $10 \%$ of the maximal load. It is possible that a decreased ability to sense force at the ankle is related to an increased risk of a sprain, which therefore would be more likely to happen toward the later stages of a match. Another aspect of proprioception that has been shown to be affected by fatigue is ankle position sense. ${ }^{32}$ Mohammadi and Roozdar $^{32}$ investigated the effects of a fatigue protocol and also the effects of 45 minutes of soccer in ankle position sense, and the results showed that both interventions had similar effects in reducing position sense in soccer players.

Another aspect previously investigated is the effect of fatigue on balance. ${ }^{12,30}$ Similar to proprioception, previous studies have shown that fatigue can negatively affect the ability to balance. ${ }^{12,30}$ Greig and McNaughton ${ }^{12}$ investigated the effect of a soccer-like exercise protocol on balance in 10 professional soccer players. 
Table 2 Characteristics of Included Studies

\begin{tabular}{|c|c|c|c|c|c|c|c|}
\hline Study & Sport & Sample & Age, y & Cohort period & $\begin{array}{l}\text { Time of } \\
\text { exposure }\end{array}$ & Total & $\begin{array}{l}\text { Ankle sprains } \\
\text { occurrence by } \\
\text { match time }\end{array}$ \\
\hline Fuller et $\mathrm{al}^{22}$ & Rugby & 941 & $19.0(0.6)$ & 4 tournaments & 166 team matches & 25 & $\begin{array}{l}\text { First quarter: } 1 \\
\text { Second quarter: } 8 \\
\text { Third quarter: } 11 \\
\text { Fourth quarter: } 5\end{array}$ \\
\hline Sankey et $\mathrm{al}^{23}$ & Rugby & 546 & NR & 2 seasons & 210 matches & 70 & $\begin{array}{l}\text { First quarter: } 10 \\
\text { Second quarter: } 22 \\
\text { Third quarter: } 9 \\
\text { Fourth quarter: } 29\end{array}$ \\
\hline Kofotolis et $\mathrm{al}^{24}$ & Amateur soccer & 312 & $24.8(4.6)$ & $2 \mathrm{y}$ & 1171 matches & 43 & $\begin{array}{l}0-15 \text { min: } 2 \\
16-30 \text { min: } 7 \\
31-45 \text { min: } 11 \\
46-60 \text { min: } 3 \\
61-75 \text { min: } 4 \\
76-90 \text { min: } 16\end{array}$ \\
\hline Carling et $\mathrm{al}^{25}$ & Soccer & $14(1$ team $)$ & NR & 6 seasons & NR & 7 & $\begin{array}{l}0-15 \text { min: } 1 \\
16-30 \text { min: } 2 \\
31-45 \text { min: } 0 \\
46-60 \text { min: } 2 \\
61-75 \text { min: } 2 \\
76-90 \text { min: } 0\end{array}$ \\
\hline Waldén et $\mathrm{al}^{26}$ & Soccer & 1743 & NR & 11 seasons & $168,952 \mathrm{~h}$ match & 285 & $\begin{array}{l}0-15 \text { min: } 32 \\
16-30 \text { min: } 48 \\
31-45 \text { min: } 55 \\
46-60 \text { min: } 53 \\
61-75 \text { min: } 53 \\
76-90 \text { min: } 44\end{array}$ \\
\hline Junge and Dvorak ${ }^{27}$ & Futsal & NR & NR & 3 world cup & 136 matches & 14 & $\begin{array}{l}\text { First quarter: } 3 \\
\text { Second quarter: } 4 \\
\text { Third quarter: } 3 \\
\text { Fourth quarter: } 4\end{array}$ \\
\hline Osbahr et $\mathrm{al}^{28}$ & American football & 1 team & NR & $15 \mathrm{y}$ & NR & 48 & $\begin{array}{l}\text { First quarter: } 8 \\
\text { Second quarter: } 14 \\
\text { Third quarter: } 16 \\
\text { Fourth quarter: } 10\end{array}$ \\
\hline O'Connor et $\mathrm{al}^{29}$ & Gaelic football & 217 (2 teams) & $19.3(1.9)$ & 1 season & $11,398 \mathrm{~h}$ & 8 & $\begin{array}{l}\text { First quarter: } 0 \\
\text { Second quarter: } 1 \\
\text { Third quarter: } 3 \\
\text { Fourth quarter: } 4\end{array}$ \\
\hline
\end{tabular}

Abbreviation: NR, not reported.

Their results showed that the center of pressure displacement increased in the mediolateral and anterior-posterior direction after exercise. Similarly, Steib et $\mathrm{al}^{30}$ reported that for 30 active athletes (14 healthy and 16 with history of ankle sprain), a fatigue protocol induced worse performance for sway velocity, "Start Excursion Balance Test," and time to stability, particularly for those with a history of ankle sprain. The findings of the studies on balance ${ }^{12,30}$ and proprioception ${ }^{31,32}$ reinforce the findings of our current systematic review, suggesting an increased risk of ankle sprain due to strenuous physical activity.
The meta-analysis by quarters showed differences in ankle sprain proportions when the first quarter was compared with the other 3 quarters. The variability in the third quarter $(95 \%$ confidence interval, 0.17-0.34) could create some doubt on whether there was a difference between the first and third quarters. Considering the level of significance found $(P=.05)$, along with clinical reasoning, it seems reasonable to consider that there was a difference in percentage of ankle sprains between the first and third quarters. It seems though that the rest period cannot be considered sufficient to provide full recovery, as there was no difference in 
Table 3 Quality Appraisal of Included Studies According to the Quality Assessment Tool for Quantitative Studies

\begin{tabular}{|c|c|c|c|c|c|c|c|}
\hline & Selection bias & Study design & Confounders & Blinding & Data collection & Withdrawals & Overall rating \\
\hline Fuller et $\mathrm{al}^{22}$ & Strong & Moderate & Strong & Moderate & Strong & Strong & Strong \\
\hline Kofotolis et $\mathrm{al}^{24}$ & Strong & Moderate & Strong & Moderate & Strong & Strong & Strong \\
\hline Junge and Dvorak ${ }^{27}$ & Strong & Moderate & Weak & Moderate & Strong & Strong & Moderate \\
\hline Osbahr et $\mathrm{al}^{28}$ & Moderate & Moderate & Weak & Moderate & Strong & N/A & Moderate \\
\hline Sankey et $\mathrm{al}^{23}$ & Strong & Moderate & Weak & Moderate & Strong & Strong & Moderate \\
\hline Waldén et $\mathrm{al}^{26}$ & Moderate & Moderate & Weak & Moderate & Strong & Weak & Weak \\
\hline Carling et $\mathrm{al}^{25}$ & Strong & Moderate & Weak & Moderate & Strong & Weak & Weak \\
\hline O'Connor et $\mathrm{al}^{29}$ & Moderate & Moderate & Weak & Weak & Strong & Strong & Weak \\
\hline
\end{tabular}

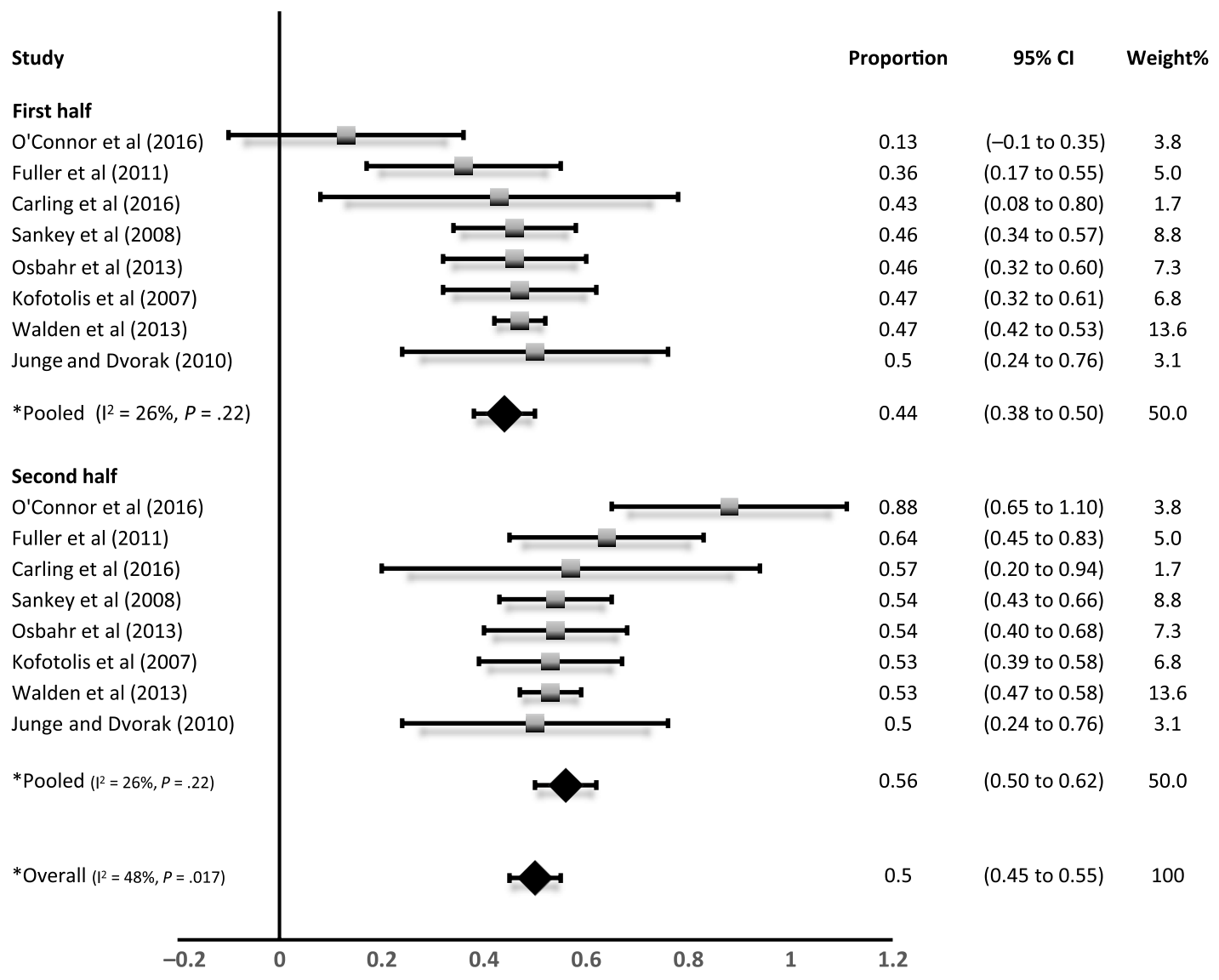

Figure 2 - Meta-analysis of proportion of ankle sprains by halftime of a game. Note: Random effects analysis.

proportions of ankle sprains between the second, third, and fourth quarters.

Although this review raises the possibility that fatigue could be a contributing factor due to the increased frequency of ankle sprains at the end of the game, it does not conclude that fatigue is the sole risk factor or cause of injury. As identified in previous studies, there are other intrinsic risk factors that also influence the incidence and recurrence of ankle sprains, such as body mass index, decreased dorsiflexion, and history of ankle sprain. ${ }^{11,33,34}$ Developing and implementing prevention strategies to reduce the impact fatigue has on an athlete may reduce the incidence of ankle sprains but will not eliminate them entirely.
The meta-analysis by sport did not replicate the results seen in the meta-analyses by halves and quarters, most likely due to a lack of power to identify such differences, as there were few studies and the number of ankle sprains by studies was also small.

One factor noted within the included studies that could have affected the results of the current systematic review is the large difference in how studies collected data on ankle sprain occurrence. Although some studies looked only at 1 single team for several seasons, others followed up with a larger number of participants for shorter periods (Table 2). Interestingly, the heterogeneity in ankle sprain proportions could be considered overall perhaps low to 


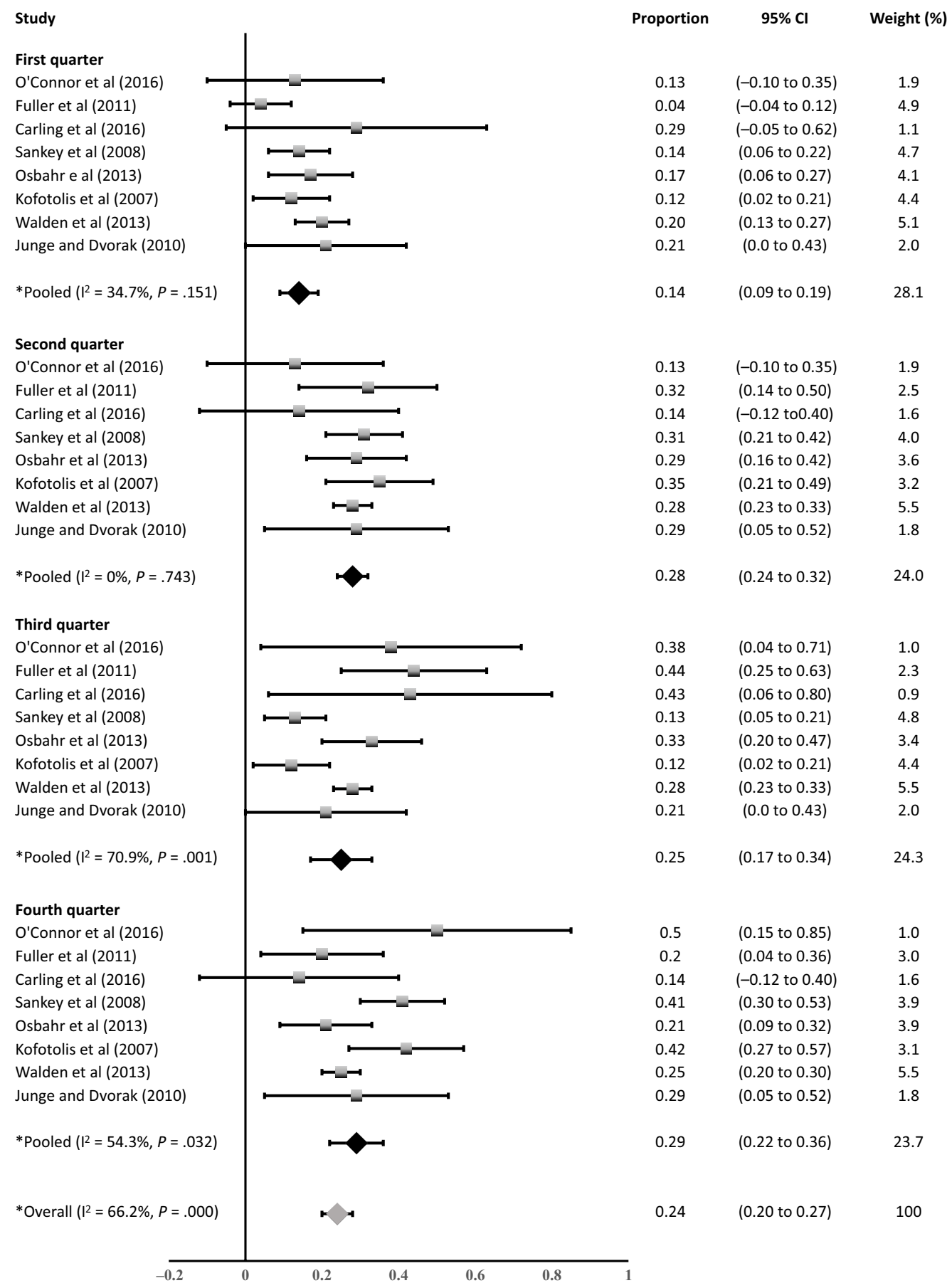

Figure 3 - Meta-analysis of proportion of ankle sprains by quarters of a game. Note: Random effects analysis.

moderate, showing that regardless of how you look at proportions of ankle sprain occurrences, the pattern seems to be similar among studies.

Currently, the information on the time ankle sprains occur during a match is limited. Although many studies (more precisely, 36) could potentially have presented specific data on ankle sprain occurrence, only 8 studies in fact did so. The studies that presented the respective data varied largely in many aspects (Table 2), such as sport, number of participants included, and time of follow up. More homogeneity in methods used among studies would likely increase credibility to the findings presented in the current review. Furthermore, the quality of the included studies varied from weak to strong, with the largest study being considered weak and potentially affecting results. However, due to the nature of the data requested for the current review, perhaps the weak characteristics of the studies that received an overall weak rating are unlikely to have a large influence on the results of this systematic review, particularly the meta-analyses.

Future research should consider exploring whether nonphysiological factors, such as change in behavior toward the end of a 
match, could help explain the pattern in ankle sprains seen in the current systematic review. Also, the time in a match when specific injuries occur, including ankle sprains, could be more frequently presented, which would increase the understanding of the pattern of these injuries.

\section{Conclusion}

Ankle sprains are more likely to happen in the second half than the first half in team sports such as rugby and soccer. Also, ankle sprain occurrence is more likely to happen toward the end of each half (second and fourth quarter) of a game. This pattern indicates that fatigue and/or exercise are likely to increase the risk of ankle sprains.

\section{References}

1. Fong DT, Hong Y, Chan LK, Yung PS, Chan KM. A systematic review on ankle injury and ankle sprain in sports. Sports Med Auckl NZ. 2007;37(1):73-94. PubMed ID: 17190537

2. Woods C, Hawkins R, Hulse M, Hodson A. The football association medical research programme: an audit of injuries in professional football: an analysis of ankle sprains. Br J Sports Med. 2003; 37(3):233-238. PubMed ID: 12782548

3. Schiftan GS, Ross LA, Hahne AJ. The effectiveness of proprioceptive training in preventing ankle sprains in sporting populations: a systematic review and meta-analysis. J Sci Med Sport. 2015; 18(3):238-244. PubMed ID: 24831756 doi:10.1016/j.jsams.2014. 04.005

4. Garrick JG, Requa RK. The epidemiology of foot and ankle injuries in sports. Clin Sports Med. 1988;7(1):29-36. PubMed ID: 2900695

5. Fong DT, Ha SC, Mok KM, Chan CW, Chan KM. Kinematics analysis of ankle inversion ligamentous sprain injuries in sports: five cases from televised tennis competitions. Am J Sports Med. 2012; 40(11):2627-2632. PubMed ID: 22967824 doi:10.1177/0363546 512458259

6. Fallat L, Grimm DJ, Saracco JA. Sprained ankle syndrome: prevalence and analysis of 639 acute injuries. J Foot Ankle Surg. 1998; 37(4):280-285. PubMed ID: 9710779

7. Andrew N, Wolfe R, Cameron P, et al. The impact of sport and active recreation injuries on physical activity levels at 12 months postinjury. Scand J Med Sci Sports. 2014;24(2):377-385. PubMed ID: 22937749 doi:10.1111/j.1600-0838.2012.01523.x

8. Doherty C, Delahunt E, Caulfield B, Hertel J, Ryan J, Bleakley C. The incidence and prevalence of ankle sprain injury: a systematic review and meta-analysis of prospective epidemiological studies. Sports Med Auckl NZ. 2014;44(1):123-140. PubMed ID: 24105612 doi:10.1007/ s40279-013-0102-5

9. Beynnon BD, Murphy DF, Alosa DM. Predictive factors for lateral ankle sprains: a literature review. J Athl Train. 2002;37(4):376-380. PubMed ID: 12937558

10. de Noronha M, França LC, Haupenthal A, Nunes GS. Intrinsic predictive factors for ankle sprain in active university students: a prospective study. Scand J Med Sci Sports. 2013;23(5):541-547. PubMed ID: 22260485 doi:10.1111/j.1600-0838.2011.01434.x

11. de Noronha M, Refshauge KM, Herbert RD, Kilbreath SL. Do voluntary strength, proprioception, range of motion, or postural sway predict occurrence of lateral ankle sprain? $\mathrm{Br}$ J Sports Med. 2006;40(10):824-828. PubMed ID: 16920769 doi:10.1136/bjsm. 2006.029645
12. Greig M, McNaughton L. Soccer-specific fatigue decreases reactive postural control with implications for ankle sprain injury. Res Sports Med Print. 2014;22(4):368-379. PubMed ID: 25295475 doi:10. 1080/15438627.2014.944300

13. Gabbett TJ. Incidence, site, and nature of injuries in amateur rugby league over three consecutive seasons. Br J Sports Med. 2000;34(2): 98-103. PubMed ID: 10786864

14. Ekstrand J, Hägglund M, Waldén M. Injury incidence and injury patterns in professional football: the UEFA injury study. Br J Sports Med. 2011;45(7):553-558. PubMed ID: 19553225 doi:10.1136/bjsm. 2009.060582

15. Murphy DF, Connolly DAJ, Beynnon BD. Risk factors for lower extremity injury: a review of the literature. Br J Sports Med. 2003; 37(1):13-29. PubMed ID: 12547739

16. Al Attar WS, Soomro N, Pappas E, Sinclair PJ, Sanders RH. How effective are F-MARC injury prevention programs for soccer players? a systematic review and meta-analysis. Sports Med Auckl NZ. 2016; 46(2):205-217. PubMed ID: 26403470 doi:10.1007/s40279-0150404-X

17. Behm DG, Blazevich AJ, Kay AD, McHugh M. Acute effects of muscle stretching on physical performance, range of motion, and injury incidence in healthy active individuals: a systematic review. Appl Physiol Nutr Metab Physiol Appl Nutr Metab. 2016;41(1):1-11. PubMed ID: 26642915 doi:10.1139/apnm-2015-0235

18. Thomas H. Quality assessment tool for quantitative studies. Toronto: Effective Public Health Practice Project McMaster University. 2003.

19. Armijo-Olivo S, Stiles CR, Hagen NA, Biondo PD, Cummings GG. Assessment of study quality for systematic reviews: a comparison of the Cochrane collaboration risk of bias tool and the effective public health practice project quality assessment tool: methodological research. J Eval Clin Pract. 2012;18(1):12-18. PubMed ID: 20698919 doi:10.1111/j.1365-2753.2010.01516.x

20. Thomas BH, Ciliska D, Dobbins M, Micucci S. A process for systematically reviewing the literature: providing the research evidence for public health nursing interventions. Worldviews Evid Based Nurs. 2004;1(3):176-184. PubMed ID: 17163895 doi:10.1111/j. 1524-475X.2004.04006.x

21. Cooper H, Hedges LV, Valentine JC, eds. The Handbook of Research Synthesis and Meta-Analysis. 2nd ed. New York, NY: Russell Sage Foundation; 2009.

22. Fuller CW, Molloy MG, Marsalli M. Epidemiological study of injuries in men's international under-20 rugby union tournaments. Clin J Sport Med. 2011;21(4):356-358. PubMed ID: 21617525 doi:10.1097/JSM.0b013e31821f5085

23. Sankey RA, Brooks JH, Kemp SP, Haddad FS. The epidemiology of ankle injuries in professional rugby union players. Am J Sports Med. 2008;36(12):2415-2424. PubMed ID: 18779364 doi:10.1177/ 0363546508322889

24. Kofotolis ND, Kellis E, Vlachopoulos SP. Ankle sprain injuries and risk factors in amateur soccer players during a 2-year period. Am J Sports Med. 2007;35(3):458-466. PubMed ID: 17218660 doi:10. $1177 / 0363546506294857$

25. Carling C, McCall A, Le Gall F, Dupont G. The impact of short periods of match congestion on injury risk and patterns in an elite football club. Br J Sports Med. 2016;50(12):764-768. PubMed ID: 26682867 doi:10.1136/bjsports-2015-095501

26. Waldén M, Hägglund M, Ekstrand J. Time-trends and circumstances surrounding ankle injuries in men's professional football: an 11-year follow-up of the UEFA champions league injury study. Br J Sports Med. 2013;47(12):748-753. PubMed ID: 23813486 doi:10.1136/ bjsports-2013-092223 
27. Junge A, Dvorak J. Injury risk of playing football in futsal world cups. Br J Sports Med. 2010;44(15):1089-1092. PubMed ID: 20961918 doi:10.1136/bjsm.2010.076752

28. Osbahr DC, Drakos MC, O'Loughlin PF, et al. Syndesmosis and lateral ankle sprains in the national football league. Orthopedics. 2013;36(11):e1378-1384. PubMed ID: 24200441 doi:10.3928/ 01477447-20131021-18

29. O’Connor S, McCaffrey N, Whyte EF, Moran KA. Epidemiology of injury in male collegiate Gaelic footballers in one season. Scand $J$ Med Sci Sports. 2017;27(10):1136-1142. PubMed ID: 27430426 doi:10.1111/sms.12733

30. Steib S, Zech A, Hentschke C, Pfeifer K. Fatigue-induced alterations of static and dynamic postural control in athletes with a history of ankle sprain. J Athl Train. 2013;48(2):203-208. PubMed ID: 23672384 doi:10.4085/1062-6050-48.1.08
31. Wright CJ, Arnold BL. Fatigue's effect on eversion force sense in individuals with and without functional ankle instability. J Sport Rehabil. 2012;21(2):127-136. PubMed ID: 22104559

32. Mohammadi F, Roozdar A. Effects of fatigue due to contraction of evertor muscles on the ankle joint position sense in male soccer players. Am J Sports Med. 2010;38(4):824-828. PubMed ID: 20139329 doi:10.1177/0363546509354056

33. Kobayashi $T$, Tanaka M, Shida M. Intrinsic risk factors of lateral ankle sprain: a systematic review and meta-analysis. Sports Health. 2016;8(2):190-193. PubMed ID: 26711693 doi:10.1177/ 1941738115623775

34. Brinkman RE, Evans TA. History of ankle sprain as a risk factor of future lateral ankle sprain in athletes. $J$ Sport Rehabil. 2011;20(3): 384-388. PubMed ID: 21828389 